\title{
Author Correction: XBP-1 and the unfolded protein response (UPR)
}

\author{
Laurie H. Glimcher (iD, Ann-Hwee Lee and Neal N. Iwakoshi (D)
}

Correction to: Nature Immunology https://doi.org/10.1038/s41590-020-0708-3, published online 2 July 2020.

In the version of this article initially published, the Competing Interests declaration was incorrect. The correct declaration is "L.H.G. is a former director of Bristol-Myers Squibb and the Waters Corporation and is currently on the board of directors of GlaxoSmithKline Pharmaceuticals and Analog Devices, Inc. She also serves on the scientific advisory boards of Repare Therapeutics, Abpro Therapeutics and Kaleido Therapeutics. A.-H.L. is an employee of Regeneron Pharmaceuticals and holds stock options in the company." The error has been corrected in the HTML and PDF versions of the article.

Published online: 12 November 2020

https://oi.org/10.1038/s41590-020-00837-6

๑ The Author(s), under exclusive licence to Springer Nature America, Inc. 2020

\section{Author Correction: A regulatory T cell Notch4-GDF15 axis licenses tissue inflammation in asthma}

Hani Harb (iD, Emmanuel Stephen-Victor, Elena Crestani, Mehdi Benamar, Amir Massoud, Ye Cui, Louis-Marie Charbonnier (D), Sena Arbag, Safa Baris (D), Amparito Cunnigham, Juan Manuel Leyva-Castillo, Raif S. Geha, Amirhosein J. Mousavi, Boris Guennewig, Klaus Schmitz-Abe, Constantinos Sioutas, Wanda Phipatanakul and Talal A. Chatila (D)

Correction to: Nature Immunology https://doi.org/10.1038/s41590-020-0777-3, published online 14 September 2020.

In the version of this article initially published, in the Methods, details were omitted from the sections "In vitro ILC2- $\mathrm{T}_{\text {reg }}$ cell co-cultures" and "In vitro ILC2-GDF15 co-cultures." In "In vitro ILC2- $\mathrm{T}_{\text {reg }}$ cell co-cultures," the cat. no. for the GDF15-blocking peptide was omitted and the concentration was incorrect. The cat. no. is MBS9229417 and the concentration was $10 \mu \mathrm{g} \mathrm{ml}^{-1}$. Additional details about the GDF15-blocking peptide omitted from this section are as follows: "The peptide was separately validated to inhibit the in vitro activation of ILC2s by E. coli-derived recombinant GDF15 (R\&D Systems, cat. no. 8944-GD), as measured by the inhibition of GDF15-induced IL-13 expression (data not shown). Seventy-two hours later, the expression of IL-13 in ILC2s was measured by flow cytometric analysis." The details omitted from the section "In vitro ILC2-GDF15 co-cultures" are as follows: "These cells were incubated with either IL-33 (10 ng ml-1), E. coli-derived recombinant GDF15 (R\&D Systems, cat. no. 8944-GD) (10 $\mu$ g ml $\left.{ }^{-1}\right)$ or both. Seventy-two hours later, the expression of IL-13 in ILC2 cells was measured by flow cytometry." The errors have been corrected in the HTML and PDF versions of the article.

Published online: 19 November 2020

https://doi.org/10.1038/s41590-020-00841-w

(c) The Author(s), under exclusive licence to Springer Nature America, Inc. 2020

\section{Author Correction: Noncanonical binding of Lck to CD3e promotes TCR signaling and CAR function}

Frederike A. Hartl, Esmeralda Beck-Garcìa, Nadine M. Woessner, Lea J. Flachsmann, Rubí M.-H. Velasco Cárdenas,

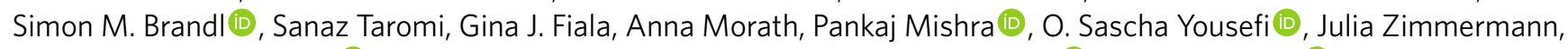
Nico Hoefflin, Maja Köhn (D), Birgitta M. Wöhrl, Robert Zeiser, Kristian Schweimer (D), Stefan Günther (D), Wolfgang W. Schamel (D) and Susana Minguet (i)

Correction to: Nature Immunology https://doi.org/10.1038/s41590-020-0732-3, published online 20 July 2020.

In the version of this article initially published, in Fig. 4, the graph in panel f duplicated the graph in panel e. The correct Fig. 4e,f are shown below. The statement about data collection and analysis at the end of the Fig. 4 caption was also incorrect. The correct statement is "Data from at least three independent experiments were pooled and analyzed using an ANOVA test." The errors have been corrected in the HTML and PDF versions of the article. 